Pacific Journal of Mathematic 


\section{TOEPLITZ FORMS AND ULTRASPHERICAL POLYNOMIALS}

JeFfrey Davis and I. I. Hirschman, JR.

For a fixed $\nu>0$ we set

$2^{n}\left(\nu+\frac{1}{2}\right)_{n} W_{\nu}(n, x)=(-1)^{n}\left(1-x^{2}\right)^{-\nu+1 / 2}\left(\frac{d}{d x}\right)^{n}\left[\left(1-x^{2}\right)^{n+\nu-1 / 2}\right]$

where $\left(\nu+\frac{1}{2}\right)_{n}=\Gamma\left(\nu+\frac{1}{2}+n\right) / \Gamma\left(\nu+\frac{1}{2}\right)$. The $W_{\nu}(n, x)$ are the ultraspherical polynomials of index $\nu$ normalized so that $W_{\nu}(n, 1)=1$. If

$$
\Omega_{\nu}(d x)=\left(1-x^{2}\right)^{\nu-1 / 2} d x, \quad \omega_{\nu}(n)=\frac{\Gamma(\nu)(n+\nu) \Gamma(n+2 \nu)}{\pi^{1 / 2} \Gamma\left(\nu+\frac{1}{2}\right) \Gamma(2 \nu) n !}
$$

then the $W_{\nu}(n, x)$ satisfy the orthogonality relations

$$
\int_{-1}^{1} W_{\nu}(n, x) W_{\nu}(m, x) \Omega_{\nu}(d x)=\left(\omega_{\nu}(n)\right)^{-1} \delta_{n, m} .
$$

Because

$$
W_{\nu}(n, x) W_{\nu}(m, x)=\sum_{k=0}^{\infty} c_{\nu}(m, n, k) W_{\nu}(k, x) \omega_{\nu}(k)
$$

where the $c_{\nu}(m, n, k)$ are nonnegative, the $\left[W_{\nu}(n, x)\right]_{n=0}^{\infty}$ behave rather like characters on a compact group. Consequently certain portions of harmonic analysis, which do not extend to orthogonal polynomials in general, have interesting analogues for ultraspherical polynomials.

In the present paper this fact is exploited to study the moments of the eigenvalues of generalized Toeplitz matrices constructed using ultraspherical polynomials.

Statement of results. Since we will always work with a fixed $\nu$ we will drop the subscript and write

$$
W_{\nu}(n, x)=W(n, x), \quad \Omega_{\nu}(d x)=\Omega(d x), \quad \omega_{\nu}(n)=\omega(n)
$$

For $f(x) \in L^{1}(\Omega)$ we set

$$
f(x) \sim \sum_{j=0}^{\infty} b(j) W(j, x)
$$

if

$$
b(j)=\omega(j) \int_{-1}^{1} f(x) W(j, x) \Omega(d x) .
$$


For $f(x) \in L^{1}(\Omega)$ let

$$
a(n, m)=[\omega(n) \omega(m)]^{1 / 2} \int_{-1}^{1} W(n, x) W(m, x) f(x) \Omega(d x) .
$$

The Toeplitz matrix of index $N$ associated with $f, A_{N}[f]$, is defined by

$$
A_{N}[f]=[a(n, m)] \quad n, m=0,1, \cdots, N .
$$

Let $[\lambda(k, N)], k=1, \cdots, N+1$, be the eigenvalues of the symmetric matrix $A_{N}[f]$. We will show that if

$$
\sum_{j=0}^{\infty}|b(j)|<\infty
$$

then

( $\left.4^{\prime}\right) \sum_{k=1}^{N+1} \lambda(k, N)^{s}=\pi^{-1}(N+1) \int_{-1}^{1} f(x)^{s}\left(1-x^{2}\right)^{2} \Omega(d x)+o(N)$ as $N \rightarrow \infty$

for $s=0,1, \cdots$. We will show further that if in addition to (4) we have

$$
\sum_{j=0}^{\infty}|b(j)|^{2} j<\infty
$$

then

$$
\begin{aligned}
\sum_{k=1}^{N+1} \lambda(k, N)^{s} & \\
= & \int_{-1}^{1} f(x)^{s}\left\{\sum_{k=0}^{N} \omega(n) W(n, x)^{2}\right\} \Omega(d x) \\
& -\sum_{j_{1}, \cdots, j_{s}=0}^{\infty} b\left(j_{1}\right) \cdots b\left(j_{s}\right) \sum_{A_{1}+\cdots+A_{s}=0} E\left(A_{1}, j_{1}\right) \cdots E\left(A_{s}, j_{s}\right) \\
& \quad \times \max \left(0, A_{1}, A_{1}+A_{2}, \cdots, A_{1}+\cdots+A_{s-1}\right)+o(1)
\end{aligned}
$$

as $N \rightarrow \infty$.

Here

$$
W(j, \cos \theta)=\sum_{A=-\infty}^{\infty} E(A, j) e^{i A \theta} .
$$

This is an analogue of a theorem on Toeplitz forms associated with Fourier series due to Kac [9].

Consider more generally

$$
f(q, r ; x)=\sum_{j=0}^{\infty} b(q, r ; j) W(j, x)
$$

where $b(q, r ; j)$ is a continuous function of $q$ and $r$ for $0 \leqq q, r \leqq 1$ for each $j=0,1, \cdots$ and where 


$$
\sum_{j=0}^{\infty} b^{\sharp}(j)<\infty, \quad b^{\sharp}(j)=\max _{q, r}|b(q, r ; j)| .
$$

Let

$$
a(n, m ; N)=[\omega(n) \omega(m)]^{1 / 2} \int_{-1}^{1} W(n, x) W(m, x) f\left(\frac{n}{N}, \frac{m}{N} ; x\right) \Omega(d x)
$$

and let

$$
A_{N}[f]=(a(n, m ; N)) \quad n, m=0,1, \cdots N .
$$

Then $A_{N}[f]$ is the "variable coefficient" Toeplitz matrix of index $N$ associated with $f(q, r ; x)$. We will show that if $(6)$ holds then

$$
\sum_{k=1}^{N+1} \lambda(k, N)^{s}=\pi^{-1}(N+1) \int_{0}^{1} \int_{-1}^{1} f(r, r ; x)^{s}\left(1-x^{2}\right)^{-\nu} \Omega(d x) d r+o(N)
$$

which is the analogue of a theorem of Kac, Murdock, and Szegö [10].

As is well known, results on moments of eigenvalues can be translated into global distribution theorems. If $f(x) \in L^{1}(\Omega)$ is real then $A_{N}[f]$ is a real symmetric matrix and then $\lambda(k, N)$ are real. Let (4) hold; if we set

$$
\alpha_{N}(E)=(N+1)^{-1} \sum_{\lambda(k, N) \in E} 1
$$

and if

$$
\alpha(E)=\pi^{-1} \int_{f(x) \in E}\left(1-x^{2}\right)^{-2} \Omega(d x)
$$

then it follows from $\left(4^{\prime}\right)$ that

$$
\alpha_{N} \longrightarrow \alpha \text { as } N \rightarrow \infty \text {. }
$$

Here $\longrightarrow$ indicates weak convergence on $(-\infty, \infty)$. This is of course a very special case of a general result due to Szegö [4]. More significantly let $b(q, r ; j)=\overline{b(r, q ; j)}$ for $0 \leqq r, q \leqq 1, j=0,1, \cdots$ and let (6) hold. Then the variable coefficient matrix (7) is Hermitian symmetric and has real eigenvalues. If $\alpha_{N}$ is the corresponding distribution function and if

$$
\beta(E)=\pi^{-1} \iint_{f(r, r ; \cos \theta) \in \epsilon_{E}} d r d \theta
$$

it follows from $\left(6^{\prime}\right)$ that

$$
\alpha_{N} \longrightarrow \beta \text { as } N \rightarrow \infty
$$

2. Properties of ultraspherical polynomials. The formulas below play a basic role in what follows. Let $k, j, n$ be nonnegative 
integers and let $2 \sigma=k+j+n$. If $k+j+n$ is even and if $\max (k, j, n) \leqq \sigma$ we set

(1) $c_{\nu}(k, j, n)$

$$
=\frac{\pi 2^{1-2 \nu}}{\Gamma(\nu)^{2}} \frac{k ! j ! n !}{(\sigma-k) !(\sigma-j) !(\sigma-n) !} \frac{(\nu)_{\sigma-k}(\nu)_{\sigma-j}(\nu)_{\sigma-n}}{(2 \nu)_{k}(2 \nu)_{j}(2 \nu)_{n}} \frac{(2 \nu)_{\sigma}}{(\nu)_{\sigma}} \frac{1}{\sigma+\nu} .
$$

$c_{\nu}(k, j, n)=0$ otherwise. Here

$$
(a)_{n}=\Gamma(a+n) / \Gamma(a) .
$$

With this definition we have

$$
\sum_{n=0}^{\infty} c_{\nu}(k, j, n) W_{\nu}(n, x) \omega_{\nu}(n)=W_{\nu}(k, x) W_{\nu}(j, x) .
$$

See Hsü [8]. We note that this series is only formally infinite since $c_{\nu}(k, j, n)=0$ if $n>k+j$. Since $W_{\nu}(n, 1)=1$ we have

$$
\sum_{n=0}^{\infty} c_{\nu}(k, j, n) \omega_{\nu}(n)=1 \text {. }
$$

Because $\nu>0$ will be fixed we now drop the subscript and write $c_{\nu}(k, j, n)=c(k, j, n)$, etc. From (2) we see that

$$
\int_{-1}^{1} W(n, x) W(m, x) W(k, x) \Omega(d x)=c(n, m, k) .
$$

Hence

$$
\begin{aligned}
& \int_{-1}^{1} W(n, x) W(m, x) W(k, x) W(p, x) \Omega(d x) \\
& \quad=\int_{-1}^{1}\left[\sum_{j=0}^{\infty} c(m, n, j) \omega(j) W(j, x)\right] W(k, x) W(p, x) \Omega(d x) \\
& \quad=\sum_{j=0}^{\infty} c(m, n, j) \omega(j) c(j, k, p) .
\end{aligned}
$$

There is no problem interchanging the integration and summation as the sums are actually finite. It follows that

(4) $W(n, x) W(m, x) W(k, x)=\sum_{p=0}^{\infty} \sum_{j=0}^{\infty} c(m, n, j) \omega(j) c(j, k, p) \omega(p) W(p, x)$.

Repeating the above argument, we find that

$$
\begin{aligned}
& \int_{-1}^{1} W\left(j_{1}, x\right) W\left(j_{2}, x\right), \cdots W\left(j_{s}, x\right) \Omega(d x) \\
& \quad=\sum_{k_{2}}, \ldots, k_{s-2}=0 \\
& c\left(j_{1}, j_{2}, k_{2}\right) \omega\left(k_{2}\right) c\left(k_{2}, j_{3}, k_{3}\right) \omega\left(k_{3}\right) \cdots \omega\left(k_{s-2}\right) c\left(k_{s-2}, j_{s-1}, j_{s}\right) .
\end{aligned}
$$

Since $\max _{-1 \leqq x \leqq 1}|W(k, x)|=W(k, 1)$, see Erdélyi [2], page 206, we have 


$$
|W(k, x)| \leqq 1 \quad k=0,1, \cdots,-1 \leqq x \leqq 1 .
$$

Let $E(h, j)$ be defined as in $\S 1$. Then

$$
\pi^{-1} \int_{-1}^{1}\left(1-x^{2}\right)^{-1 / 2} W(2 j, x) d x=E(0,2 j) .
$$

This is because

$$
\begin{aligned}
\pi^{-1} \int_{-1}^{1}\left(1-x^{2}\right)^{-1 / 2} W(2 j, x) d x & =\pi^{-1} \int_{0}^{\pi} W(2 j, \cos \theta) d \theta \\
& =\pi^{-1} \int_{0}^{\pi}\left\{\sum_{-2 j}^{2 j} E(h, 2 j) e^{i h \theta}\right\} d \theta \\
& =(2 \pi)^{-1} \int_{0}^{2 \pi}\left\{\sum_{-2 j}^{2 j} E(h, 2 j) e^{i h \theta}\right\} d \theta \\
& =E(0,2 j) .
\end{aligned}
$$

Here we have used the fact that $E(h, 2 j)$ is 0 if $h$ is odd.

We will have occasion to use the following inequality,

$$
\left|\omega(k)^{1 / 2}(\sin \theta)^{\nu} W(k, \cos \theta)\right| \leqq c(\nu),
$$

which is given in Szegö [12; §7.32].

Using asymptotic estimates for Jacobi and ultraspherical polynomials, see Szegö [12; 8.21$]$, and adjusting for our normalization we obtain

(9) $W_{\nu}(k, \cos \theta)$

$$
=2 \frac{\Gamma(2 \nu) \Gamma(k+\nu)}{\Gamma(\nu) \Gamma(k+2 \nu)}\left\{\frac{\cos [(k+\nu) \theta-\nu \pi / 2]+(k \sin \theta)^{-1} \theta(1)}{(2 \sin \theta)^{\nu}}\right\}
$$

for $\delta k^{-1} \leqq \theta \leqq \pi-\delta k^{-1}$ (here $0(1)$ is uniform in $\theta$ and $k$ if $\delta>0$ is fixed) and

$$
\begin{aligned}
W_{\nu}(n, \cos \theta)= & 2 \frac{\Gamma(2 \nu) \Gamma(n+\nu)}{\Gamma(\nu) \Gamma(n+2 \nu)}\left\{\frac{\cos [(n+\nu) \theta-\nu \pi / 2]}{(2 \sin \theta)^{\nu}}\right. \\
& \left.-\frac{\nu(\nu-1)}{(n+\nu-1)} \frac{\sin [(n+\nu-1) \theta-\nu \pi / 2]}{(2 \sin \theta)^{\nu+1}}\right\} \\
& +0\left(n^{-\nu-3 / 2}\right)
\end{aligned}
$$

for $\theta$ fixed, $0<\theta<\pi$.

We will have occasion to use the following asymptotic formula

$$
\begin{aligned}
& \frac{\Gamma\left(z+\alpha_{1}\right) \Gamma\left(z+\alpha_{2}\right) \cdots \Gamma\left(z+\alpha_{r}\right)}{\Gamma\left(z+\beta_{1}\right) \Gamma\left(z+\beta_{2}\right) \cdots \Gamma\left(z+\beta_{r}\right)} \\
& \quad=1+(2 z)^{-1}\left(\sum_{n=1}^{r} \alpha_{n}^{2}-\beta_{n}^{2}\right)+0\left(z^{-2}\left[\sum_{n}^{r}\left|\alpha_{n}\right|^{3}+\left|\beta_{n}\right|^{3}\right]\right),
\end{aligned}
$$

as $|z| \rightarrow \infty$ where $\alpha_{1}+\alpha_{2}+\cdots+\alpha_{r}=\beta_{1}+\beta_{2}+\cdots+\beta_{r},|\arg z|<\pi$. See Erdélyi [2], page 47. 
We further note

$$
\frac{d}{d x} W_{\nu}(n, x)=\frac{n(n+2 \nu)}{2 \nu+1} W_{\nu+1}(n-1, x) .
$$

We conclude this section by deriving the following limit relation.

THEOREM 2a. With the notations of $\S 1$ if $k, k^{\prime}$ and $j$ are fixed integers, $j \geqq 0$, then

$$
\lim _{N \rightarrow \infty} \omega(N+k) c\left(N+k, j, N+k^{\prime}\right)=E\left(k-k^{\prime}, j\right) .
$$

Proof. Let

$$
I_{N}=\left[\omega(N+k) \omega\left(N+k^{\prime}\right)\right]^{1 / 2} c\left(N+k, j, N+k^{\prime}\right) .
$$

Then

$$
\begin{aligned}
I_{N}= & \int_{0}^{\pi}\left\{(\omega(N+k))^{1 / 2} W(N+k, \cos \theta) \sin ^{\nu} \theta\right\} \\
& \times\left\{\left(\omega\left(N+k^{\prime}\right)\right)^{1 / 2} W\left(N+k^{\prime}, \cos \theta\right) \sin ^{\nu} \theta\right\} W(j, \cos \theta) d \theta .
\end{aligned}
$$

We know, see (8) and (9), that

$$
\begin{aligned}
& \lim _{N \rightarrow \infty}\left\{(\omega(N))^{1 / 2} W(N, \cos \theta) \sin ^{\nu} \theta-(2 / \pi)^{1 / 2} \cos [(N+\nu) \theta-\pi \nu / 2]\right\}=0 \\
& 0<\theta<\pi
\end{aligned}
$$

and that there is a constant $c(\nu)$ such that

$$
\left|(\omega(N))^{1 / 2} W(N, \cos \theta) \sin ^{\nu} \theta\right| \leqq c(\nu) \quad N=0,1, \cdots, 0 \leqq \theta \leqq \pi .
$$

Using the Lebesgue limit theorem we see that

$$
\begin{aligned}
\lim _{N \rightarrow \infty} I_{N}= & \lim _{N \rightarrow \infty} 2 \pi^{-1} \\
& \times \int_{0}^{\pi} \cos [(N+k+\nu) \theta-\pi \nu / 2] \cos \left[\left(N+k^{\prime}+\nu\right) \theta-\pi \nu / 2\right] W(j, \cos \theta) d \theta
\end{aligned}
$$

if the limit on the right exists. We have

$$
\begin{aligned}
& \cos [(N+k+\nu) \theta-\pi \nu / 2] \cos \left[\left(N+k^{\prime}+\nu\right) \theta-\pi \nu / 2\right] \\
& \quad=\frac{1}{2} \cos \left[\left(k-k^{\prime}\right) \theta\right]+\frac{1}{2} \cos \left[\left(2 N+k+k^{\prime}+2 \nu\right) \theta-\pi \nu\right] .
\end{aligned}
$$

By the Riemann-Lebesgue theorem

$$
\lim _{N \rightarrow \infty} \pi^{-1} \int_{0}^{\pi} \cos \left[\left(2 N+k+k^{\prime}+2 \nu\right) \theta-\pi \nu\right] W(j, \cos \theta) d \theta=0
$$

while 


$$
\pi^{-1} \int_{0}^{\pi} \cos \left[\left(k-k^{\prime}\right) \theta\right] W(j, \cos \theta) d \theta=E\left(k-k^{\prime}, j\right) .
$$

Finally, it is easily checked that

$$
\lim _{N \leftarrow \infty}\left[\frac{\omega(N+k)}{\omega\left(N+k^{\prime}\right)}\right]^{1 / 2}=1 .
$$

Combining these facts we have our desired result.

3. Two basic limit relations.

THEOREM 3a. With the notations of $\S 1$ we have

$$
\left|(\sin \theta)^{2 \nu} \sum_{k=0}^{n} \omega(k) W_{\nu}^{2}(k, \cos \theta)-\pi^{-1}(n+1)\right| \leqq A(\nu)(\sin \theta)^{-2}
$$

for $0 \leqq \theta \leqq \pi, n=0,1, \cdots$.

Proof. Set

$$
S_{n}(x)=\sum_{k=0}^{n} \omega(k) W(k, x)^{2} .
$$

By the Christoffel-Darboux formula, see Erdélyi [3] page 159, we get

$$
\begin{aligned}
S_{n}(x)= & \frac{1}{\pi} \frac{1}{2^{2-2 \nu}}\left(\frac{\Gamma(\nu)}{\Gamma(2 \nu)}\right)^{2} \frac{\Gamma(n+2 \nu+1)}{\Gamma(n+1)}\left[W_{\nu}^{\prime}(n+1, x) W_{\nu}(n, x)\right. \\
& \left.-W_{\nu}^{\prime}(n, x) W_{\nu}(n+1, x)\right] .
\end{aligned}
$$

Upon substituting (12) $\S 2$, the above becomes

$$
\begin{aligned}
S_{n}(x)= & \frac{1}{\pi} \frac{2^{2 \nu-2}}{2 \nu+1}\left(\frac{\Gamma(\nu)}{\Gamma(2 \nu)}\right)^{2} \frac{\Gamma(n+2 \nu+1)}{\Gamma(n+1)} \\
& \times\left[(n+1)(n+2 \nu+1) W_{\nu+1}(n, x) W_{\nu}(n, x)\right. \\
& \left.-n(n+2 \nu) W_{\nu+1}(n-1, x) W_{\nu}(n+1, x)\right] .
\end{aligned}
$$

Now using the estimate (9) $\S 2$ with $\delta=1$ we obtain

$$
(\sin \theta)^{2 \nu} S_{n}(\cos \theta)=\frac{1}{\pi}\left\{(n+1)+(\sin \theta)^{-2} O(1)\right\} \quad n^{-1}<\theta<\pi-n^{-1} .
$$

On the other hand it follows from (8) $\S 2$ that

$$
(\sin \theta)^{2 \nu} S_{n}(\cos \theta)=O(n) \quad 0 \leqq \theta \leqq \pi .
$$

Combining these results we obtain the desired inequality. 


$$
\lim _{N \rightarrow \infty} \int_{-1}^{1}\left|\pi^{-1}\left(1-x^{2}\right)^{-\nu}-(N+1)^{-1} \sum_{k=0}^{\infty} \omega(k) W(k, x)^{2}\right| \Omega(d x)=0 .
$$

Proof. This follows from the above together with the relations

$$
\begin{gathered}
\pi^{-1} \int_{-1}^{1}\left(1-x^{2}\right)^{-\nu} \Omega(d x)=1, \\
\int_{-1}^{1}(N+1)^{-1} \sum_{k=0}^{N} \omega(k) W(k, x)^{2} \Omega(d x)=1 .
\end{gathered}
$$

This result implies that if $f \in L^{\infty}[-1,1]$ then

$$
\int_{-1}^{1}\left\{\sum_{k=0}^{n} \omega_{\nu}(k) W_{\nu}(k, x)^{2}\right\} f(x) \Omega_{\nu}(d x)=\frac{n+1}{\pi} \int_{-1}^{1}\left(1-x^{2}\right)^{-1 / 2} f(x) d x+o(n)
$$

as $n \rightarrow \infty$. The following gives a more precise evaluation, but for a slightly more restricted class of functions $f$.

THEOREM 3c. Let $f(x)$ be bounded for $-1 \leqq x \leqq 1$ and let $[f(1)-f(x)](1-x)^{-3 / 2}$ and $[f(x)-f(-1)](1+x)^{-3 / 2}$ be integrable. Then

$$
\begin{aligned}
\int_{-1}^{1}\left\{\sum_{k=0}^{n} \omega_{\nu}(k) W_{\nu}(k, x)^{2}\right\} f(x) \Omega_{\nu}(d x) & \\
= & \frac{n+1}{\pi} \int_{-1}^{1} f(x)\left(1-x^{2}\right)^{-1 / 2} d x+\frac{2 \nu-1}{2 \pi} \int_{-1}^{1} f(x)\left(1-x^{2}\right)^{-1 / 2} d x \\
& \quad-\frac{2 \nu-1}{4}[f(1)+f(-1)]+o(1),
\end{aligned}
$$

as $n \rightarrow \infty$.

Proof. Let

$$
f_{1}(x)=\frac{1}{2}[f(x)+f(-x)]-\frac{1}{2}[f(1)+f(-1)] .
$$

A simple even-odd argument together with (2) shows that, using the notation of Theorem 3a,

$$
\int_{-1}^{1} S_{n}(x) f(x) \Omega(d x)=\int_{-1}^{1} S_{n}(x) f_{1}(x) \Omega(d x)+\frac{n+1}{2}[f(1)+f(-1)] .
$$

It is therefore sufficient to determine the asymptotic behavior of

$$
\int_{-1}^{1} S_{n}(x) f_{1}(x) \Omega(d x)=\int_{0}^{\pi}(\sin \theta)^{2 \nu} S_{n}(\cos \theta) f_{1}(\cos \theta) d \theta .
$$

We will show that as $n \rightarrow \infty$ 
( 3 )

$$
\begin{aligned}
& \int_{0}^{\pi}(\sin \theta)^{2 \nu} S_{n}(\cos \theta) f_{1}(\cos \theta) d \theta \\
& \quad=\frac{1}{\pi}\left[(n+1)+\frac{2 \nu-1}{2}\right] \int_{0}^{\pi} f_{1}(\cos \theta) d \theta+o(1) .
\end{aligned}
$$

Let us assume for the moment that (3) has been demonstrated. Then

$$
\begin{aligned}
\int_{-1}^{1} S_{n}(x) f(x) \Omega(d x) \\
=\frac{1}{\pi}\left[n+1+\frac{2 \nu-1}{2}\right] \int_{-1}^{1} f_{1}(x)\left(1-x^{2}\right)^{-1 / 2} d x+\frac{n+1}{2}[f(1)+f(-1)]+o(1), \\
=\frac{1}{\pi}\left[(n+1)+\frac{2 \nu-1}{2}\right] \int_{-1}^{1}\left\{f(x)-\frac{1}{2}[f(1)+f(-1)]\right\}\left(1-x^{2}\right)^{-1 / 2} d x \\
\quad+\frac{n+1}{2}[f(1)+f(-1)]+o(1), \\
=\frac{n+1}{\pi} \int_{-1}^{1} f(x)\left(1-x^{2}\right)^{-1 / 2} d x+\frac{2 \nu-1}{2 \pi} \int_{-1}^{1} f(x)\left(1-x^{2}\right)^{-1 / 2} d x \\
\quad-\frac{2 \nu-1}{4}[f(1)+f(-1)]+o(1),
\end{aligned}
$$

as desired. It thus remains only to demonstrate (3). Our assumptions imply that

$$
\int_{-1}^{1}\left|f_{1}(\cos \theta)\right| \sin ^{-2} \theta d \theta<\infty
$$

We assert that, if $0<\theta<\pi$,

( 5 ) $\lim _{n \rightarrow \infty}\left\{(\sin \theta)^{2 \nu} S_{n}(\cos \theta)\right.$

$$
\left.-\frac{1}{\pi}\left[(n+1)+\frac{2 \nu-1}{2}+\frac{\sin (2 n+2 \nu+1) \theta-\nu \pi}{2 \sin \theta}\right]\right\}=0 .
$$

It is evident that Theorem 3a, (4) and (5) together imply (3). Here we use the Riemann-Lebesgue lemma in order to dispose of the term which arises from the $\sin [(2 n+2 \nu+1) \theta-\nu \pi] / 2 \sin \theta$ on the right in (5).

By (10) Section 2 we see that for $\theta$ fixed, $0<\theta<\pi$,

$$
\begin{aligned}
W_{\nu}(n, \cos \theta)= & 2 \frac{\Gamma(2 \nu)}{\Gamma(\nu)} \frac{\Gamma(n+\nu)}{\Gamma(n+2 \nu)}\left[\frac{\cos \left[(n+\nu) \theta-\frac{\nu \pi}{2}\right]}{(2 \sin \theta)^{\nu}}\right. \\
& \left.-\frac{\nu(\nu-1)}{(n+\nu-1)} \frac{\sin \left[(n+\nu-1) \theta-\frac{\nu \pi}{2}\right]}{(2 \sin \theta)^{\nu+1}}\right]+O\left(n^{-\nu-\frac{3}{2}}\right),
\end{aligned}
$$




$$
\begin{aligned}
W_{\nu+1}(n, \cos \theta)= & 4(2 \nu+1) \frac{\Gamma(2 \nu)}{\Gamma(\nu)} \frac{\Gamma(n+\nu+1)}{\Gamma(n+2 \nu+2)}\left[\frac{\sin \left[(n+\nu+1) \theta-\frac{\nu \pi}{2}\right]}{(2 \sin \theta)^{\nu+1}}\right. \\
& \left.+\frac{(\nu+1) \nu}{(n+\nu)} \frac{\cos \left[(n+\nu) \theta-\frac{\nu \pi}{2}\right]}{(2 \sin \theta)^{\nu+2}}\right]+O\left(n^{\left.-\nu-\frac{5}{2}\right)},\right. \\
W_{\nu}(n+1, \cos \theta)= & 2 \frac{\Gamma(2 \nu)}{\Gamma(\nu)} \frac{\Gamma(n+\nu+1)}{\Gamma(n+2 \nu+1)}\left[\frac{\cos \left[(n+\nu+1) \theta-\frac{\nu \pi}{2}\right]}{(2 \sin \theta)^{\nu}}\right. \\
& \left.-\frac{\nu(\nu-1)}{(n+\nu)} \frac{\sin \left[(n+\nu) \theta-\frac{\nu \pi}{2}\right]}{(2 \sin \theta)^{\nu+1}}\right]+O\left(n^{\left.-\nu-\frac{3}{2}\right)},\right. \\
W_{\nu+1}(n-1, \cos \theta)= & 4(2 \nu+1) \frac{\Gamma(2 \nu)}{\Gamma(\nu)} \frac{\Gamma(n+\nu)}{\Gamma(n+2 \nu+1)}\left[\frac{\sin \left[(n+\nu) \theta-\frac{\nu \pi}{2}\right]}{(2 \sin \theta)^{\nu+1}}\right. \\
& +\frac{\nu(\nu+1)}{(n+\nu-1)} \frac{\cos \left[(n+\nu-1) \theta-\frac{\nu \pi}{2}\right]}{(2 \sin \theta)^{\nu+2}}
\end{aligned}
$$

Substituting into (1) we obtain

$(\sin \theta)^{2 \nu} S_{n}(\cos \theta)$

$$
\begin{aligned}
& =\frac{1}{\pi} \frac{\Gamma(n+\nu) \Gamma(n+\nu+1)}{\Gamma(n+1) \Gamma(n+2 \nu)} \\
& \times\left\{( n + 1 ) \left[\frac{\cos \left[(n+\nu) \theta-\frac{\nu \pi}{2}\right] \sin \left[(n+\nu+1) \theta-\frac{\nu \pi}{2}\right]}{\sin \theta}\right.\right. \\
& +\frac{\nu(\nu+1)}{2(n+\nu)} \frac{\left(\cos \left[(n+\nu) \theta-\frac{\nu \pi}{2}\right]\right)^{2}}{(\sin \theta)^{2}} \\
& \left.-\frac{\nu(\nu-1)}{2(n+\nu-1)} \frac{\sin \left[(n+\nu-1) \theta-\frac{\nu \pi}{2}\right] \sin \left[(n+\nu+1) \theta-\frac{\nu \pi}{2}\right]}{(\sin \theta)^{2}}\right] \\
& -n\left[\frac{\cos \left[(n+\nu-1) \theta-\frac{\nu \pi}{2}\right] \sin \left[(n+\nu) \theta-\frac{\nu \pi}{2}\right]}{\sin \theta}\right. \\
& +\frac{\nu(\nu+1)}{2(n+\nu-1)} \frac{\cos \left[(n+\nu-1) \theta-\frac{\nu \pi}{2}\right] \cos \left[(n+\nu+1) \theta-\frac{\nu \pi}{2}\right]}{(\sin \theta)^{2}} \\
& \left.\left.-\frac{\nu(\nu-1)}{2(n+\nu)} \frac{\left(\sin \left[(n+\nu) \theta-\frac{\nu \pi}{2}\right]\right)^{2}}{(\sin \theta)^{2}}\right]\right\}+O\left(n^{-1 / 2}\right) \text {. }
\end{aligned}
$$


Simplification gives

$(\sin \theta)^{2 \nu} S_{n}(\cos \theta)$

$$
\begin{aligned}
= & \frac{1}{\pi} \frac{\Gamma(n+\nu) \Gamma(n+\nu+1)}{\Gamma(n+1) \Gamma(n+2 \nu)}\left[(n+1)+\nu^{2}-\frac{1}{2}+\frac{\sin \mid(2 n+2 \nu+1) \theta-\nu \pi]}{2 \sin \theta}\right] \\
& +O\left(n^{-1 / 2}\right) .
\end{aligned}
$$

Now, from (11) Section 2,

$$
\frac{\Gamma(n+\nu) \Gamma(n+\nu+1)}{\Gamma(n+1) \Gamma(n+2 \nu)}=1+\frac{\nu-\nu^{2}}{n+1}+O\left(n^{-2}\right),
$$

and hence, if $0<\theta<\pi$,

$$
\begin{aligned}
(\sin \theta)^{2 \nu} S_{n}(\cos \theta)= & \frac{1}{\pi}\left[(n+1)+\frac{2 \nu-1}{2}+\frac{\sin [(2 n+2 \nu+1) \theta-\nu \pi]}{2 \sin \theta}\right] \\
& +O\left(n^{-1 / 2}\right)
\end{aligned}
$$

where the $O\left(n^{-1 / 2}\right)$ depends upon $\theta$. Our proof is now complete.

4. First order approximation of moments. As in $\S 1$ let

$$
f(x)=\sum_{j=0}^{\infty} b(j) W(j, x)
$$

where

$$
\sum_{0}^{\infty}|b(j)|<\infty
$$

Since $|W(j, x)| \leqq 1$ by (6) of $\S 2$ it follows that the series defining $f(x)$ converges absolutely and uniformly for $-1 \leqq x \leqq 1$ so that $f(x)$ is a continuous function on $-1 \leqq x \leqq 1$. Let

$$
a(n, m)=[\omega(n), \omega(m)]^{1 / 2} \int_{-1}^{1} W(n, x) W(m, x) f(x) \Omega(d x)
$$

and let $\{\lambda(k, N)\}, k=1,2, \cdots, N+1$ be the eigenvalues of the Toeplitz matrix $A_{N}[f]=[a(n, m)], n, m=0,1, \cdots, N$.

THEOREM 4a. Under the above assumptions

$$
\begin{array}{r}
\sum_{k=1}^{N+1}(\lambda(k, N))^{s}=\pi^{-1}(N+1) \int_{-1}^{1}(f(x))^{s}\left(1-x^{2}\right)^{-1 / 2} d x+o(N) \\
\text { as } N \rightarrow \infty .
\end{array}
$$

for $s=0,1, \cdots$.

Proof. Note that 


$$
\sum_{k=1}^{N+1}(\lambda(k, N))^{s}=\operatorname{tr}\left(A_{N}[f]\right)^{s} .
$$

Substituting (1) in the definition of $a(n, m)$ we get

$$
a(n, m)=\sum_{j=0}^{\infty} b(j) c(n, j, m)
$$

where the $c(n, j, m)$ are defined as in $\S 2$. Consequently

( 5 ) $\operatorname{tr} A_{N}[f]^{s}=\sum_{k_{1}, k_{2}}, \sum_{, k_{s}=0}^{N} a\left(k_{1}, k_{2}\right) a\left(k_{2}, k_{3}\right) \cdots a\left(k_{s}, k_{1}\right)$

$$
\begin{aligned}
= & \sum_{j_{1}}, \cdots, j_{s}=0 \\
& \times \sum_{k_{1}, \cdots, k_{s}=0}^{\infty} \omega\left(j_{1}\right) b\left(j_{2}\right) \cdots b\left(k_{1}, j_{1}, k_{2}\right) \omega\left(k_{2}\right) \cdots \omega\left(k_{s}\right) c\left(k_{s}, j_{s}, k_{1}\right) .
\end{aligned}
$$

Since $c(k, j, l)$ vanishes unless $2 \max (j, k, l) \leqq j+k+l$ the above sums are all finite. Let

$$
K(s, N)=\int_{-1}^{1} f(x)^{s}\left\{\sum_{k=0}^{N} \omega(k)(W(k, x))^{2}\right\} \Omega(d x) .
$$

We have

$$
\begin{aligned}
K(s, N)= & \int_{-1}^{1}\left\{\sum_{j=0}^{\infty} b(j) W(j, x)\right\}^{s}\left\{\sum_{k_{1}=0}^{N} \omega\left(k_{1}\right)\left(W\left(k_{1}, x\right)\right)^{2}\right\} \Omega(d x), \\
= & \sum_{j_{1}, \cdots, j_{s}=0}^{\infty} b\left(j_{1}\right) \cdots b\left(j_{s}\right) \\
& \times \sum_{k_{1}=0}^{N} \int_{-1}^{1} W\left(k_{1}, x\right) W\left(j_{1}, x\right) \cdots W\left(j_{s}, x\right) W\left(k_{1}, x\right) \Omega(d x) \\
= & \sum_{j_{1}, \cdots, j_{s}=0}^{\infty} b\left(j_{1}\right) \cdots b\left(j_{s}\right) \\
& \times \sum_{k_{1}=0}^{N} \sum_{k_{2}, \cdots, k_{s}=0}^{\infty} \omega\left(k_{1}\right) c\left(k_{1}, j_{1}, k_{2}\right) \cdots \omega\left(k_{s}\right) c\left(k_{s}, j_{s}, k_{1}\right) .
\end{aligned}
$$

Here we have used formula (5) from $§ 2$. Comparing (5) and (6) we see that

$$
\operatorname{tr}\left(A_{N}[f]\right)^{s}-K(s, N)=-\sum_{j_{1}, \cdots, j_{s}}^{\infty} b\left(j_{1}\right) \cdots b\left(j_{s}\right) \psi(j, N)
$$

where $\boldsymbol{j}=\left(j_{1}, \cdots, j_{s}\right)$ and

( 7) $\psi(j, N)=\sum_{Q \backslash N)} \omega\left(k_{1}\right) c\left(k_{1}, j_{1}, k_{2}\right) \omega\left(k_{2}\right) c\left(k_{2}, j_{2}, k_{3}\right) \cdots \omega\left(k_{s}\right) c\left(k_{s}, j_{s}, k_{1}\right)$.

Here $Q(N)$ consists of all $s$-tuples $\left(k_{1}, \cdots, k_{s}\right)$ for which $0 \leqq k_{1} \leqq N$, $0 \leqq k_{\alpha} \leqq \infty, \alpha=2,3, \cdots, s$, and $k_{\alpha}>N$ for at least one $\alpha=2, \cdots, s$.

It follows from (3) of $\S 2$ that $0 \leqq \omega\left(k_{1}\right) c\left(k_{1}, j_{1}, k_{2}\right) \leqq 1$. Replacing $\omega\left(k_{1}\right) c\left(k_{1}, j_{1}, k_{2}\right)$ by 1 in $(7)$ and summing over $0 \leqq k_{1} \leqq N, 0 \leqq k_{\alpha}<\infty$, 
$\alpha=2,3, \cdots, s$ we find that

$$
0 \leqq \psi(j, N) \leqq N+1
$$

On the other hand since $c\left(k_{\alpha}, j_{\alpha}, k_{\alpha+1}\right)=0$ if $\left|k_{\alpha}-k_{\alpha+1}\right|>j_{\alpha}, \alpha=1$, $\cdots, s-1$, the fact that some $k_{\alpha}>N$ implies that we may assume

$$
N-\left(j_{1}+\cdots+j_{s-1}\right) \leqq k_{1} \leqq N
$$

since otherwise the summand in (7) vanishes. Repeating the argument above gives

$$
0 \leqq \psi(j, N) \leqq j_{1}+\cdots+j_{s-1} .
$$

Using (8) we see that

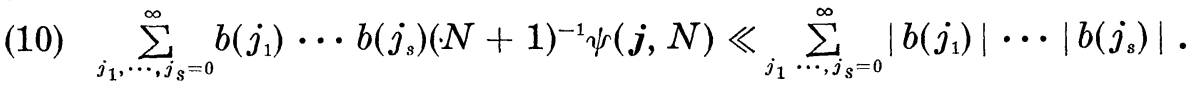

As a consequence of (2) the sum of the series on the right here is finite. On the other hand using (9) we have

$$
\lim _{N \rightarrow \infty}(N+1)^{-1} \psi(j, N)=0 .
$$

The relations (10) and (11) together imply that

$$
\lim _{N \rightarrow \infty}(N+1)^{-1}\left\{\operatorname{tr}\left[A_{N}[f]^{s}\right]-K(s, N)\right\}=0 .
$$

Finally it follows from Corollary $3 \mathrm{~b}$ that

$$
\begin{aligned}
\lim _{N \rightarrow \infty}(N+1)^{-1} K(s, N) & =\lim _{N \rightarrow \infty} \int_{-1}^{1} f(x)^{s}(N+1)^{-1}\left\{\sum_{0}^{N} \omega(k)(W(k, x))^{2}\right\} \Omega(d x) \\
& =\pi^{-1} \int_{-1}^{1} f(x)^{s}\left(1-x^{2}\right)^{-1 / 2} d x
\end{aligned}
$$

and we are done.

Let $f(x)$ be real and Riemann in integrable on $[-1,1]$ and let (as in $\S 1)$

$$
\begin{aligned}
\alpha_{N}(E) & =(N+1)^{-1} \sum_{\lambda(k, N) \in \mathbb{B}} 1, \\
\alpha(E) & =\pi^{-1} \sum_{f(x) \in E_{B}}\left(1-x^{2}\right)^{-1 / 2} d x .
\end{aligned}
$$

Then it follows from (4) by a standard argument, see [4], that

$$
\alpha_{N}(E) \longrightarrow \alpha(E) \quad \text { as } N \rightarrow \infty \text {. }
$$

Conversely (12) implies (4). Results like (12) hold in much greater generality. Our excuse for the inclusion of Theorem $4 \mathrm{a}$ is that its demonstration shows in a simple setting the basic idea of the present paper. 
5. Second order approximation of moments. Let us now assume that

$$
f(x)=\sum_{j=0}^{\infty} b(j) W(j, x)
$$

where

$$
M_{1}=\sum_{j=0}^{\infty}|b(j)|<\infty, \quad M_{2}=\left(\sum_{j=0}^{\infty} j|b(j)|^{2}\right)^{1 / 2}<\infty .
$$

THEOREM 5a. Under the above assumptions we have

$$
\begin{aligned}
& \sum_{k=0}^{N} \lambda(k, N)^{s} \\
& =\int_{-1}^{1} f(x)^{s}\left\{\sum_{0}^{N} \omega(k)(W(k, x))^{2}\right\} \Omega(d x) \\
& \quad-\sum_{j_{1}, \cdots, j_{s}=0}^{\infty} b\left(j_{1}\right) \cdots b\left(j_{s}\right) \sum_{h_{1}+\cdots+h_{s}=0} E\left(h_{1}, j_{1}\right) \cdots E\left(h_{s}, j_{s}\right) \\
& \quad \times \max \left(0, h_{1}, h_{1}+h_{2}, \cdots, h_{1}+\cdots+h_{s-1}\right)+o(1)
\end{aligned}
$$

for $s=0,1, \cdots$, and where the $E(h, j)$ are defined by

$$
W(j, \cos \theta)=\sum_{h=-\infty}^{\infty} E(h, j) e^{i n \theta} .
$$

Proof. The relation (3) is exact for $s=0,1$. We therefore suppose $s \geqq 2$. As in the proof of Theorem 4 a we have

$$
\operatorname{tr}\left(A_{N}[f]^{s}\right)-K(s, N)=-\sum_{j_{1}}, \sum_{, j, j_{s}=0}^{\infty} b\left(j_{1}\right) \cdots b\left(j_{s}\right) \psi(j, N) .
$$

Let us set $h_{1}=k_{2}-k_{1}, h_{2}=k_{3}-k_{2}, \cdots, h_{s-1}=k_{s}-k_{s-1}, h_{s}=k_{s}-k_{1}$. If $\left|h_{1}\right|>j_{1}$ then $c\left(k_{1}, j_{1}, k_{2}\right)=0$ etc. so that all the terms in $Q(N)$ which do not give zero are included in those for which $\left|h_{\alpha}\right| \leqq j_{\alpha}$, $\alpha=1, \cdots, s$. Moreover since $k_{\alpha}=k_{1}+h_{1}+h_{2}+\cdots+h_{\alpha-1}$ we see that as $k_{\alpha}>N$ for some $\alpha$ we must have

$$
k_{1}+\max \left(0, h_{1}, h_{1}+h_{2}, \cdots, h_{1}+\cdots+h_{s-1}\right)>N .
$$

Thus by a very crude estimate

$$
N-\sum_{\alpha=1}^{8}\left|h_{\alpha}\right| \leqq k_{1} \leqq N .
$$

Since $h_{1}+h_{2}+\cdots+h_{s}=0$

$$
\sum_{\alpha=1}^{s}\left|h_{\alpha}\right| \leqq \sum_{\substack{\alpha=1 \\ \alpha \neq \beta}}^{s}\left|h_{\alpha}\right|^{1 / 2}\left|h_{\beta}\right|^{1 / 2} \leqq \sum_{\substack{\alpha, \beta=1 \\ \alpha \neq \beta}}^{s}\left|j_{\alpha}\right|^{1 / 2}\left|j_{\beta}\right|^{1 / 2}
$$


If we replace $\omega\left(k_{1}\right) c\left(k_{1}, j_{1}, k_{2}\right)$ by 1 in (7) Section 4 and if we replace $Q(N)$ by all $\left(k_{1}, \cdots, k_{s}\right)$ for which

$$
N-\sum_{\substack{\alpha, \beta=1 \\ \alpha \neq \beta}}^{s}\left|j_{\alpha}\right|^{1 / 2}\left|j_{\beta}\right|^{1 / 2} \leqq k_{1} \leqq N, \quad 0 \leqq k_{\alpha} \leqq \infty, \alpha=2, \cdots, s,
$$

we find that

$$
0 \leqq \psi(j, N) \leqq \sum_{\substack{\alpha, \beta=1 \\ \alpha \neq \beta}}^{s}\left|j_{\alpha}\right|^{1 / 2}\left|j_{\beta}\right|^{1 / 2}
$$

Using

$$
\left|b\left(j_{\alpha}\right) b\left(j_{\beta}\right)\right|\left|j_{\alpha}\right|^{1 / 2}\left|j_{\beta}\right|^{1 / 2} \leqq \frac{1}{2}\left|b\left(j_{\alpha}\right)\right|^{2}\left|j_{\alpha}\right|+\frac{1}{2}\left|b\left(j_{\beta}\right)\right|^{2}\left|j_{\beta}\right|
$$

we find that for $s \geqq 2$

$$
\sum_{j_{1}, \cdots, j_{s}} \mid b\left(j_{1}\right) \cdots\left(\left.b\left(j_{s}\right)\left|\sum_{\substack{\alpha, \beta=1 \\ \alpha \neq \beta}}^{s}\right| j_{\alpha}\right|^{1 / 2}\left|j_{\beta}\right|^{1 / 2} \leqq s^{2} M_{2}^{2} M_{1}^{s-2} .\right.
$$

Returning to $\psi(j, N)$ let $k=k_{1}-N$. Then

$$
\begin{aligned}
\psi(j, N)= & \sum \omega(k+N) c\left(k+N, j_{1}, k+N+h_{1}\right) \\
& \times \omega\left(k+N+h_{1}, j_{2}, k+N+h_{1}+h_{2}\right) \cdots \omega\left(k+N+h_{1}+\cdots+h_{s-1}\right) \\
& \times c\left(k+N+h_{1}+\cdots+h_{s-1}, j_{s}, k+N\right),
\end{aligned}
$$

where the summation is extended over those indices $k, h_{1}, \cdots, h_{s-1}$ for which

$$
\begin{aligned}
& 0 \geqq k \geqq-\max \left(0, h_{1}, h_{1}+h_{2}, \cdots, h_{1}+\cdots+h_{s-1}\right), \\
& 0 \geqq k \geqq-N .
\end{aligned}
$$

Note however that unless the indices $k, h_{1}, \cdots, h_{s-1}$ satisfy the conditions

$$
\begin{aligned}
& 0 \geqq k \geqq-\sum_{\substack{\alpha, \beta=1 \\
\alpha \neq \beta}}^{s}\left|j_{\alpha}\right|^{1 / 2}\left|j_{\beta}\right|^{1 / 2}, \\
& \left|h_{\alpha}\right| \leqq j_{\alpha}, \alpha=1, \cdots, s-1 .
\end{aligned}
$$

the corresponding term in the sum above well be 0 . For $j_{1}, \cdots, j_{s}$ fixed the restriction $0 \geqq k \geqq-N$ becomes otiose for large $N$. It follows from Theorem $2 \mathrm{a}$ that

$$
\lim _{N \rightarrow \infty} \omega(N+k) c\left(N+k, j_{1}, N+k+h_{1}\right)=E\left(h_{1}, j_{1}\right)
$$

etc., which implies that

$$
\lim _{N \rightarrow \infty} \psi(j, N)=\sum_{k, h_{1}, \cdots, h_{s}} E\left(h_{1}, j_{1}\right) \cdots E\left(h_{s}, j_{s}\right)
$$


the summation being extended over $h_{1}, \cdots, h_{s}$ subject to the restriction $h_{1}+\cdots+h_{s}=0$ and over $k$ satisfying the first condition of (9). Thus

$$
\begin{aligned}
\lim _{N \rightarrow \infty} \psi(j, N)= & \sum_{h_{1}+} \sum_{++h_{s}=0} E\left(h_{1}, j_{1}\right) \cdots E\left(h_{s}, j_{s}\right) \\
& \times \max \left(0, h_{1}, h_{1}+h_{2}, \cdots, h_{1}+\cdots+h_{s-1}\right) .
\end{aligned}
$$

We have previously shown that

$$
\sum_{j_{1}, \cdots, j_{s}=0}^{\infty} b\left(j_{1}\right) \cdots b\left(j_{s}\right) \psi(j, N) \ll \sum_{j_{1}, \cdots, j_{s}}^{\infty}\left|b\left(j_{1}\right) \cdots b\left(j_{s}\right)\right| \sum_{\substack{\alpha, \beta=1 \\ \alpha \neq \beta}}^{s}\left|j_{\alpha}\right|^{1 / 2}\left|j_{\beta}\right|^{1 / 2}
$$

where the series on the right is convergent (its sum not exceeding $s^{2} M_{1}^{s-2} M_{2}^{2}$ ). The relations (11) and (12) together clearly give (3).

Making use of Theorem $3 \mathrm{c}$ we obtain the following more explicit result.

Corollary 5b. If in addition to (1) and (2) it is assumed that $[f(x)-f(1)](1-x)^{-3 / 2}$ and $[f(x)-f(-1)](x+1)^{-3 / 2}$ are integrable then for $s=0,1, \cdots$

$$
\begin{aligned}
\sum_{k=0}^{N} \lambda(k, N)^{s}= & \frac{N+1}{\pi} \int_{-1}^{1} f(x)^{s}\left(1-x^{2}\right)^{-1 / 2} d x+\frac{2 \nu-1}{\pi} \int_{-1}^{1} f(x)^{s}\left(1-x^{2}\right)^{-1 / 2} d x \\
- & \frac{2 \nu-1}{4}\left[f(1)^{s}+f(-1)^{s}\right]-\sum_{j_{1}, \cdots, j_{s}=0}^{\infty} b\left(j_{1}\right) \cdots b\left(j_{s}\right) \\
& \times \sum_{h_{1}+\cdots+h_{s}=0} E\left(h_{1}, j_{1}\right) \cdots E\left(h_{s}, j_{s}\right) \\
& \times \max \left(0, h_{1}, \cdots, h_{1}+\cdots+h_{s-1}\right)+o(1) \text { as } N \rightarrow \infty .
\end{aligned}
$$

We will now, following a method due to Kac [9], use Theorem 5a to study the asymptotic behavior of

$$
D_{N}[f]=\operatorname{det}\left[A_{N}[f]\right]=\lambda(1, N) \cdots \lambda(N+1, N) .
$$

We define

$$
\log G_{i N}[f]=\int_{-1}^{1}[\log f(x)](N+1)^{-1}\left\{\sum_{k=0}^{N} \omega(k)(W(k, x))^{2}\right\} \Omega(d x) .
$$

Let $\rho$ be any complex number satisfying $|\rho|\|f\|_{\infty}<1$ and let $F(x)=$ $1-\rho f(x)$. Then

$$
\begin{aligned}
D_{N}[F] & =\prod_{k=1}^{N+1}[1-\rho \lambda(k, N)], \\
\log D_{N}[F] & =\sum_{k=1}^{N+1} \log [1-\rho \lambda(k, N)], \\
& =\sum_{k=1}^{N+1} \sum_{\alpha=1}^{\infty} \frac{-\rho^{\alpha}(\lambda(k, N))^{\alpha}}{\alpha}, \\
& =\sum_{\alpha=1}^{\infty} \frac{-\rho^{\alpha}}{\alpha} \operatorname{tr}\left(A_{N}[f]^{\alpha}\right) .
\end{aligned}
$$


Similarly, using the notation of Section 4,

$$
\begin{aligned}
\log G_{N}[F] & =\int_{-1}^{1} \log [1-\rho f(x)](N+1)^{-1}\left\{\sum_{k=0}^{N} \omega(k)(W(k, x))^{2}\right\} \Omega(d x), \\
& =\int_{-1}^{1}\left\{\sum_{\alpha=1}^{\infty} \frac{-\rho^{\alpha}(f(x))^{\alpha}}{\alpha}\right\}(N+1)^{-1}\left\{\sum_{k=0}^{N} \omega(k)(W(k, x))^{2}\right\} \Omega(d x), \\
& =(N+1)^{-1} \sum_{\alpha=1}^{\infty} \frac{-\rho^{\alpha}}{\alpha} K(\alpha, N) .
\end{aligned}
$$

Thus

$$
\log \left\{\frac{D_{N}[f]}{G_{N}[F]^{N+1}}\right\}=\sum_{\alpha=1}^{\infty} \frac{-\rho^{\alpha}}{\alpha}\left\{\operatorname{tr}\left(A_{N}[f]\right)^{\alpha}-K(\alpha, N)\right\} .
$$

It follows from (8) that

$$
\left|\operatorname{tr}\left(A_{N}[f]\right)^{\alpha}-K(\alpha, N)\right| \leqq \alpha^{2} M_{1}^{\alpha-2} M_{2} \quad \alpha \geqq 2
$$

uniformly in $N$. Using (14) and (3) we see that if $|\rho| M_{1}<1$ then

(15) $\lim _{N \rightarrow \infty} \log \left\{\frac{D_{N}[F]}{G_{N}[F]^{N+1}}\right\}$

$$
\begin{aligned}
= & -\sum_{\alpha=1}^{\infty} \frac{-\rho^{\alpha}}{\alpha} \sum_{j_{1}, \cdots j_{\alpha}=0} b\left(j_{1}\right) \cdots b\left(j_{\alpha}\right) \sum_{h_{1}+\cdots+h_{\alpha}=0} E\left(h_{1}, j_{1}\right) \cdots E\left(h_{\alpha}, j_{\alpha}\right) \\
& \times \max \left(0, h_{1}, h_{1}+h_{2}, \cdots, h_{1}+\cdots+h_{\alpha-1}\right) .
\end{aligned}
$$

Consider

$$
\begin{aligned}
f(\cos \theta) & =\sum_{j=0}^{\infty} b(j) W(j, \cos \theta) \\
& =\sum_{j=0}^{\infty} b(j) \sum_{h=-\infty}^{\infty} E(h, j) e^{i h \theta} \\
& =\sum_{h=-\infty}^{\infty} c(h) e^{i h \theta}
\end{aligned}
$$

where

$$
c(h)=\sum_{j=0}^{\infty} b(j) E(h, j) .
$$

It follows that the right hand side of (15) is equal to $-\sum_{\alpha=1}^{\infty} \frac{-\rho^{\alpha}}{\alpha} \sum_{h_{1}+\cdots+h_{\alpha}=0} c\left(h_{1}\right) \cdots c\left(h_{\alpha}\right) \max \left(0, h_{1}, h_{1}+h_{2}, \cdots, h_{1}+\cdots+h_{\alpha-1}\right)$. We have

$$
E(h, j) \geqq 0 ;
$$

see [2, Vol, II, p. 175]. Since 


$$
W(j, \cos \theta)=\sum_{h=-j}^{j} E(h, j) e^{i h \theta}
$$

it follows on setting $\theta=0$ that

$$
1=\sum_{h=-j}^{j} E(h, j) \text {. }
$$

From this one sees that if $f(x)$ is given by (1) and if $M_{1}$ and $M_{2}$ are finite then

$$
\sum_{-\infty}^{\infty}|c(h)|<\infty, \quad \sum_{-\infty}^{\infty}|c(h)|^{2}|h|<\infty .
$$

It follows that as a consequence of an important combinatorial identity discovered by Kac in [9] and later studied by Spitzer and others (a particularly accessible reference is [14]), the right hand side of (15) can be written as

$$
\frac{1}{2} \sum_{n=1}^{\infty} B_{\lambda}(n) B_{\lambda}(-n) n
$$

where

$$
\log F(\cos \theta)=\sum_{-\infty}^{\infty} B_{\lambda}(n) e^{i n \theta} .
$$

In these connections see [1].

We have thus shown that if $|\rho| M_{1}<1$ then

$$
\lim _{N \rightarrow \infty} \frac{D_{N}[F]}{G_{N}[F]^{N+1}}=\exp \left[\frac{1}{2} \sum_{n=1}^{\infty} B_{\lambda}(n) B_{\lambda}(-n) n\right] .
$$

A moments thought shows that this result can be rephrased as follows.

THEOREM 5b. Let $f(x)=\sum_{0}^{\infty} b(j) W_{\nu}(j, x)$ satisfy conditions (1) and (2) and suppose in addition that

$$
|b(0)|>\sum_{1}^{\infty}|b(j)|
$$

Then

$$
\lim _{N \rightarrow \infty} \frac{D_{N}[f]}{G_{N}[f]^{N+1}}=\exp \left[\frac{1}{2} \sum_{n=1}^{\infty} b_{\lambda}(n) b_{\lambda}(-n) n\right]
$$

where

$$
\log f(\cos \theta) \sim \sum_{-\infty}^{\infty} b_{\lambda}(n) e^{i n \theta}
$$


It is clear that if in addition $f$ satisfies the assumptions of Theorem $3 \mathrm{c}$ then this can be written in the simpler form

$$
\lim _{N \rightarrow \infty} \frac{D_{N}\lceil f\rceil}{G\left\lceil\left. f\right|^{N+1+(2 \nu+1) / 2}\right.}=[f(1)-f(-1)]^{-(2 \nu-1) / 4} \exp \left[\frac{1}{2} \sum_{n=1}^{\infty} b_{\lambda}(n) b_{\lambda}(-n) n\right],
$$

where

$$
G[f]=\exp \left[\frac{1}{\pi} \int_{-1}^{1} \log f(x)\left(1-x^{2}\right)^{-1 / 2} d x\right] .
$$

It will be shown by one of us in a subsequent paper that

$$
|b(0)|>\sum_{1}^{\infty}|b(j)|
$$

can be replaced by the weaker condition

$$
f(x)=0 \quad-1 \leqq x \leqq 1 .
$$

We thus obtain a complete analogue for Toeplitz forms associated with ultraspherical polynomials of the best version of the strong Szegö limit theorem for (ordinary) Toeplitz forms. See [13] and |7|. Theorem 5b is an essential step in the demonstration of this result.

\section{Matrices with variable coefficients.}

LeMma 6a. Let $p(r)$ be continuous on $0 \leqq r \leqq 1$. Then

$$
\lim _{N \rightarrow \infty}(N+1)^{-1} \sum_{k=0}^{N}(\omega)(k) W(k, x)^{2} \uparrow\left(\frac{k}{N}\right)=\pi^{-1}\left(1-x^{2}\right)^{-2} \int_{a}^{1} \mathfrak{P}(r) d r,
$$

for each $x,-1<x<1$.

Proof. Let $A_{N}$ be the measure on $[0,1]$ whose mass is concentrated at the points $k N^{-1}, k=0, \cdots, N$ and for which

$$
\Lambda_{N}\left(\left\{k N^{-1}\right\}\right)=(N+1)^{-1} \omega(k) W(k, x)^{2} .
$$

Then

$$
(N+1)^{-1} \sum_{k=0}^{N} \omega(k) W(k, x)^{2} \mathfrak{P}\left(\frac{k}{N}\right)=\int_{0}^{1} \mathfrak{\rho}\left(r^{\circ}\right) \Lambda_{N}\left(d r^{*}\right) .
$$

Let $(a, b) \subseteq[0,1] ;$ then

$$
\begin{aligned}
A_{N}[(a, b)]= & \left.(N+1)^{-1} \sum_{a<k} \sum_{N^{-1}} \omega\right)(k) W(k, x)^{\circ} \\
= & b\lceil(N+1) b\rceil^{-1} \sum_{0<k_{k<b_{N}}}(o)(k) W(k, x)^{\circ} \\
& -a\lceil(N+1) a\rceil^{-1} \sum_{0<k<a N} \omega(k) W(k, x)^{\circ},
\end{aligned}
$$




$$
\cong(b-a) \pi^{-1}\left(1-x^{2}\right)^{-\nu} \text { as } N \rightarrow \infty .
$$

by Corollary $3 \mathrm{~b}$. The same relation persists if $(a, b)$ is replaced by $(a, b]$, etc. Our result is an easy consequence of this.

Let

$$
f(q, r ; x)=\sum_{j=0}^{\infty} b(q, r ; j) W(j, x)
$$

be a complex valued function defined for $0 \leqq q, r \leqq 1,-1 \leqq x \leqq 1$. We assume that the following conditions are satisfied:

A.

$$
b(q, r ; j) \text { is continuous for } 0 \leqq q, r \leqq 1 \text { for each } j=0,1, \cdots \text {; }
$$

$$
\sum_{j=0}^{\infty} b^{\sharp}(j)=M<\infty \quad \text { where } \quad b^{\sharp}(j)=\max _{q, r}|b(q, r ; j)| .
$$

Since $|W(j, x)| \leqq 1$ by (6), $\S 2$, A. implies that the series defining $f(q, r ; x)$ converges absolutely and uniformly for $-1 \leqq x \leqq 1$ and hence $f(q, r ; x)$ is continuous in all variables.

We form the matrix $A_{N}[f]=(a(n, k ; N) n, k=0,1, \cdots, N$, where

$$
a(n, k ; N)=[\omega(n) \omega(k)]^{1 / 2} \int_{-1}^{1} W(n, x) W(k, x) f\left(\frac{n}{N}, \frac{k}{N} ; x\right) \Omega(d x) .
$$

THEOREM 6b. With the above definitions if $f$ satisfies conditions A. we have

$$
\lim _{N \rightarrow \infty}(N+1)^{-1} \operatorname{tr}\left(A_{N}[f]^{s}\right)=\pi^{-1} \int_{-1}^{1} \int_{0}^{1} f(r, r ; x)^{s}\left(1-x^{2}\right)^{-1 / 2} d r d x
$$

Proof. First, as is easily seen from the formulae of $\S 2$, we have

$$
a(n, k ; N)=\sum_{j=0}^{\infty} \omega(k) c(k, n, j) b\left(\frac{n}{N}, \frac{k}{N} ; j\right) .
$$

Using this, a straightforward computation gives

$$
(N+1)^{-1} \operatorname{tr}\left(A_{N}[f]^{s}\right)=I(s, N)
$$

where

$$
\begin{aligned}
I(s, N)= & (N+1)^{-1} \sum_{j_{1}, \cdots, j_{s}=0}^{\infty} \sum_{k_{1}}^{N}, \cdots, k_{s}=0 \\
& \times b\left(\frac{k_{1}}{N}, \frac{k_{2}}{N} ; j_{1}\right) b\left(\frac{k_{2}}{N}, \frac{k_{3}}{N} ; j_{2}\right) \cdots b\left(\frac{k_{s}}{N}, \frac{k_{1}}{N} ; j_{s}\right) \psi(k, j) .
\end{aligned}
$$

Here,

$$
\begin{aligned}
\psi(\boldsymbol{k}, \boldsymbol{j}) & =\omega\left(k_{1}\right) c\left(k_{1}, j_{1}, k_{2}\right) \omega\left(k_{2}\right) c\left(k_{2}, j_{2}, k_{3}\right) \cdots \omega\left(k_{s}\right) c\left(k_{s}, j_{s}, k_{1}\right), \\
\boldsymbol{k} & =\left(k_{1}, \cdots, k_{s}\right), \boldsymbol{j}=\left(j_{1}, \cdots, j_{s}\right) .
\end{aligned}
$$


Let

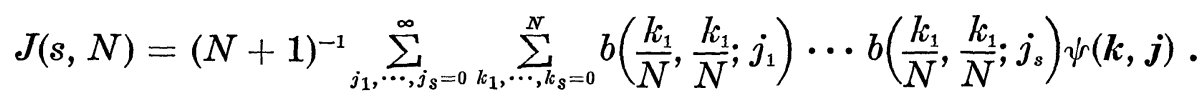

We wish to show that

$$
\lim _{N \rightarrow \infty}[I(s, N)-J(s, N)]=0 .
$$

We have

$$
I(s, N)-J(s, N)=\sum_{j_{1}, \cdots, j_{s}=0}^{\infty} d(j, s, N)
$$

where

$$
\begin{aligned}
d(\boldsymbol{j}, s, N)= & (N+1)^{-1} \sum_{k_{1}, \cdots, k_{s}=0}^{N}\left\{b\left(\frac{k_{1}}{N}, \frac{k_{2}}{N} ; j_{1}\right) \cdots b\left(\frac{k_{s}}{N}, \frac{k_{1}}{N} ; j_{s}\right)\right. \\
& \left.-b\left(\frac{k_{1}}{N}, \frac{k_{1}}{N} ; j_{1}\right) \cdots b\left(\frac{k_{1}}{N}, \frac{k_{1}}{N} ; j_{s}\right)\right\} \psi(\boldsymbol{k}, \boldsymbol{j}) .
\end{aligned}
$$

We need only consider terms for which $\left|k_{\alpha}-k_{\alpha-1}\right| \leqq j_{\alpha-1}, \alpha=2, \cdots, s$, since if this condition is violated $\psi(k, j)=0$. Using the uniform continuity of $b(q, r ; j)$ for $j=0,1, \cdots$ we see that

$$
\begin{aligned}
\mid b\left(\frac{k_{1}}{N}, \frac{k_{2}}{N} ; j_{1}\right) \cdots b\left(\frac{k_{s}}{N}, \frac{k_{1}}{N} ; j_{s}\right) & -b\left(\frac{k_{1}}{N}, \frac{k_{1}}{N} ; j_{1}\right) \cdots b\left(\frac{k_{1}}{N}, \frac{k_{1}}{N} ; j_{s}\right) \mid \\
& \leqq 2 b^{\sharp}\left(j_{1}\right) \cdots b^{\sharp}\left(j_{s}\right) \eta(j, s, N)
\end{aligned}
$$

where

$$
\begin{aligned}
& |\eta(j, s, N)| \leqq 1, \\
& \lim _{N \rightarrow \infty} \eta(j, s, N)=0 .
\end{aligned}
$$

Thus, by a now familiar argument,

$$
\begin{aligned}
|d(\boldsymbol{j}, s, N)| & \leqq 2 b^{\sharp}\left(j_{1}\right) \cdots b^{\sharp}\left(j_{s}\right) \eta(\boldsymbol{j}, s, N)(N+1)^{-1} \sum_{k_{1}, \ldots, k_{s}=0}^{W} \psi(\boldsymbol{k}, \boldsymbol{j}), \\
& \leqq 2 b^{\sharp}\left(j_{1}\right) \cdots b^{\sharp}\left(j_{s}\right) \eta(j, s, N) .
\end{aligned}
$$

Taken together these facts imply (1).

We next consider

$$
K(s)=\pi^{-1} \int_{1}^{1} \int_{0}^{1} f(r, r ; x)^{s}\left(1-x^{2}\right)^{-\nu} d r \Omega(d x) .
$$

By Lemma 6a

$$
\pi^{-1} \int_{0}^{1} f(r, r ; x)^{s}\left(1-x^{2}\right)^{-\nu} d r=\lim _{N \rightarrow \infty}(N+1)^{-1} \sum_{k=0}^{N} \omega(k) W(k, x)^{2}\left[f\left(\frac{k}{N}, \frac{k}{N} ; x\right)\right]^{s}
$$


for each $x,-1<x<1$. Since $f$ is bounded and since by (8) of $\S 2$

$$
(N+1)^{-1} \sum_{k=0}^{N} \omega(k) W(k, x)^{2} \leqq C(\nu)\left(1-x^{2}\right)^{-\nu}
$$

the Lebesgue convergence theorem can be applied to show that

$$
K(s)=\lim _{N \rightarrow \infty} K(s, N)
$$

where

$$
\begin{aligned}
& K(s, N)=(N+1)^{-1} \sum_{k=0}^{N} \int_{-1}^{1} f\left(\frac{k}{N}, \frac{k}{N} ; x\right)^{s} \omega(k) W(k, x)^{2} \Omega(d x),
\end{aligned}
$$

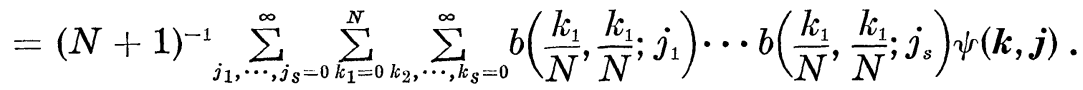

We have

$$
|K(s, N)-J(s, N)| \leqq(N+1)^{-1} \sum_{j_{1}, \cdots, j_{s}=0}^{\infty} b^{\sharp}\left(j_{1}\right) \cdots b^{\sharp}\left(j_{s}\right) \psi(j, N) .
$$

Since, see (8) and (9) of $\S 4$,

$$
\begin{aligned}
& (N+1)^{-1} \psi(j, N) \leqq 1, \\
& \lim _{N \rightarrow \infty}(N+1)^{-1} \psi(j, N)=0,
\end{aligned}
$$

it follows that

$$
\lim _{N \rightarrow \infty}[K(s, N)-J(s, N)]=0 .
$$

The relations (1), (2) and (3) combined yield our theorem.

THEOREM 6c. Let $f$ satisfy conditions A. and let $f(q, r ; x)=$ $\overline{f(r, q ; x)}$ for $0 \leqq r, q \leqq 1,-1 \leqq x \leqq 1$. If $\{\lambda(N, k)\}_{k=1}^{N+1}$ are the (necessarily real) eigenvalues of $A_{N}(f)$ and if

$$
\begin{aligned}
\alpha_{N}(E) & =(N+1)^{-1} \sum_{\lambda(k, N) \in E} 1, \\
\beta(E) & =\pi^{-1} \iint_{f(r, r ; \cos \theta) \in E} d r d \theta,
\end{aligned}
$$

then

$$
\alpha_{N} \longrightarrow \beta \text { as } N \rightarrow \infty \text {. }
$$

Proof. We first note that

$$
\operatorname{tr}\left(A_{N}[f]^{s}\right)=\sum_{k=1}^{N+1} \lambda(k, N)^{s} .
$$


From Theorem $6 \mathrm{~b}$ we have the convergence of the moments and the proof then follows standard lines. See [4], page 98.

For other results connected with Toeplitz matrices of the kind considered here see [6].

7. Second order approximation in the case of variable coefficients. Let $A_{N}[f]=[a(m, n ; N)], n=0, \cdots, N$ be defined as in $\S 6$. Under certain, fairly restrictive assumptions we can show that, as $N \rightarrow \infty$,

$$
\begin{aligned}
& \operatorname{tr}\left[A_{N}[f]^{s}\right] \\
& -\int_{-1}^{1} \sum_{k=0}^{N} f\left(\frac{k}{N}, \frac{k}{N} ; x\right)^{s} \omega(k) W(k, x)^{2} \Omega(d x) \\
& =-\sum_{j_{1}, \cdots, j_{s}=0}^{\infty} b\left(1,1 ; j_{1}\right) \cdots b\left(1,1 ; j_{s}\right) \sum_{h_{1}+\cdots+h_{s}=0} E\left(h_{1}, j_{1}\right) \cdots E\left(h_{s}, j_{s}\right) \\
& \quad \times \max \left(0, h_{1}, h_{1}+h_{2}, \cdots, h_{1}+\cdots+h_{s-1}\right)+o(1) .
\end{aligned}
$$

This is the analogue of a theorem of Schmidt and Mejlbo [11]. Since the demonstration of (1) is rather long and awkward it has seemed best to us to omit it.

\section{BIBLIOGRAPHY}

1. Dunford and Schwartz, Linear Operators, Part I, Interscience, New York, 1958.

2. Erdélyi et al., Higher Transcendental Functions, Vol. I, New York, 1953.

3. - Higher Transcendental Functions, Vol. 11, New York, 1953.

4. U. Grenander and G. Szegö, Toeplitz forms and their applications, Berkeley and Los Angeles, 1958.

5. I. I. Hirschman, Jr., Variation diminishing transformations and ultraspherical polynomials, Jour. d'Anal. Math. 8 (1960-61), 337-359.

6. - Extreme eigen values of Toeplitz forms associated with ultraspherical polynomials, Jour. of Math. and Mech. 13 (1964), 249-282.

7. - On a theorem of Szegö, Kac and Baxter (to appear in Jour. d'Analyse Math.)

8. H.-Y. Hsu, Certain integrals and infinite series involving ultraspherical polynomials and Bessel functions, Duke Math. J. 4 (1938), 374-383.

9. M. Kac, Toeplitz matrices, translation kernels and a related problem in probability theory, Duke Math. J. 21 (1954), 501-9.

10. M. Kac, W. Murdock and G. Szegö, On the eigenvalues of certain hermitian forms, J. Rat. Mech. Anal. 2 (1953), 767-800.

11. L. Mejlbo and P. Schmidt, On the eigenvalues of generalized Toeplitz matrices, Math. Scand. 10 (1962), 5-16.

12. G. Szegö, Orthogonal polynomials, Amer. Math. Soc. Col. Publ. 23, New York, 1959. 13. - On certain Hermitian forms associated with the Fourier series of a positive function, Commun. du seminaire math de l'Univ. de Lund, tome supp. (1952), 228-237.

14. J. G. Wendel, Spitzer's formula: a short proof, Proc. Amer. Math. Soc. 9 (1958), 905-908.

Received March 11, 1965. Research supported in part by the United States Air Force Office of Scientific Research under Contract No. AF-AFOSR 721-65. 



\section{PACIFIC JOURNAL OF MATHEMATICS}

H. SAMELSON

Stanford University Stanford, California

R. M. BLUMENTHAL

University of Washington

Seattle, Washington 98105

\section{EDITORS}

\author{
*J. DugundJI \\ University of Southern California \\ Los Angeles, California 90007 \\ RICHARD ARENS \\ University of California \\ Los Angeles, California 90024
}

\section{ASSOCIATE EDITORS}

E. F. BeCKENBACH

B. H. NEUMANN

F. WOLF

K. YosidA

\section{SUPPORTING INSTITUTIONS}

UNIVERSITY OF BRITISH COLUMBIA

CALIFORNIA INSTITUTE OF TECHNOLOGY

UNIVERSITY OF CALIFORNIA

MONTANA STATE UNIVERSITY

UNIVERSITY OF NEVADA

NEW MEXICO STATE UNIVERSITY

OREGON STATE UNIVERSITY

UNIVERSITY OF OREGON

OSAKA UNIVERSITY

UNIVERSITY OF SOUTHERN CALIFORNIA
STANFORD UNIVERSITY

UNIVERSITY OF TOKYO

UNIVERSITY OF UTAH

WASHINGTON STATE UNIVERSITY

UNIVERSITY OF WASHINGTON

AMERICAN MATHEMATICAL SOCIETY CHEVRON RESEARCH CORPORATION TRW SYSTEMS

NAVAL ORDNANCE TEST STATION 


\section{Pacific Journal of Mathematics}

Vol. 18, No. 1

March, 1966

Edward Joseph Barbeau, Semi-algebras that are lower semi-lattices ...... 1

Steven Fredrick Bauman, The Klein group as an automorphism group

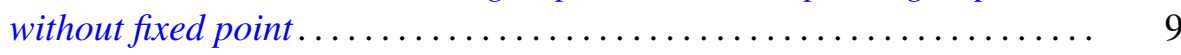

Homer Franklin Bechtell, Jr., Frattini subgroups and $\Phi$-central groups .... 15

Edward Kenneth Blum, A convergent gradient procedure in prehilbert

spaces ............................................

Edward Martin Bolger, The sum of two independent exponential-type random variables ...................................

David Wilson Bressler and A. P. Morse, Images of measurable sets .......

Dennison Robert Brown and J. G. LaTorre, A characterization of uniquely

divisible commutative semigroups........................

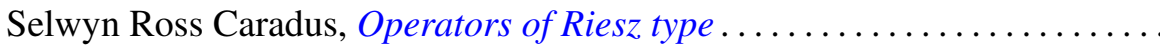

Jeffrey Davis and Isidore Isaac Hirschman, Jr., Toeplitz forms and ultraspherical polynomials ............................

Lorraine L. Foster, On the characteristic roots of the product of certain rational integral matrices of order two ......................

Alfred Gray and S. M. Shah, Asymptotic values of a holomorphic function

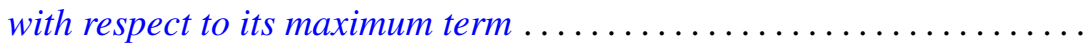

Sidney (Denny) L. Gulick, Commutativity and ideals in the biduals of

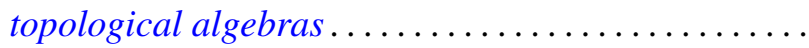

G. J. Kurowski, Further results in the theory of monodiffric functions

Lawrence S. Levy, Commutative rings whose homomorphic images are self-injective .

Calvin T. Long, On real numbers having normality of order $k$....

Bertram Mond, An inequality for operators in a Hilbert space. ...

John William Neuberger, The lack of self-adjointness in three-point boundary value problems ........................

C. A. Persinger, Subsets of $n$-books in $E^{3}$

Oscar S. Rothaus and John Griggs Thompson, A combinatorial problem in the symmetric group ............................... 175

Rodolfo DeSapio, Unknotting spheres via Smale .................. 179

James E. Shockley, On the functional equation

$$
F(m n) F((m, n))=F(m) F(n) f((m, n)) \ldots \ldots \ldots
$$

Kenneth Edward Whipple, Cauchy sequences in Moore spaces ... 